\title{
Radar Recognition Using Pulse Repetition Interval Technology
}

\author{
Imran Ahmad ${ }^{1}$, Mohammed S BinMelha ${ }^{1,2}$, Ahmed S.I. Amar ${ }^{1,3}$, Mohammed J. Ngala ${ }^{4}$, Raed Abd- \\ Alhameed ${ }^{2,5}$ \\ I.ahmad3@bradford.ac.uk, abofaras@yahoo.com, a.a.s.ismail@bradford.ac.uk, mjngala@ heatoned.co.uk, \\ r.a.a.abd@bradford.ac.uk
}

\author{
${ }^{1}$ Faculty of engineering and informatics, University of Bradford, Bradford, BD7 1DP, UK. \\ ${ }^{2}$ System Maintenance Controller, Saudi Air Navigation Services, KSA \\ ${ }^{3}$ Faculty of Engineering, Ain Shams University, Cairo, Egypt \\ ${ }^{4}$ Heaton Education Bradford UK \\ ${ }^{4}$ Information and Communication Eng. Department, Basrah University College of Science and \\ Technology, Basrah 24001, Iraq
}

\begin{abstract}
Recognition of pulse repetition interval modulation (PRI) of a radar signal is important. It helps in identifying the radar's functional purpose and assists in emitter identification. This paper discusses two methods, the gate time pulse method and feature extraction, used for PRI modulation recognition. These features are based on both sequential and statistical information of the pulse intervals. The extracted features can be used for emitter recognition by feeding the information in a neural network.
\end{abstract}

Keywords: RPI modulation, PRF, Radar Recognition, Variable PRI emitters, Fixed PRI emitter Location Based Services, LBS, Smart City, Emitter recognition, Radar recognition, Pulse Repetition Interval, Signals Intelligence, PRF.

\section{Introduction}

Advances in the radar technology result in the usage of complicated radar waveforms. The radar signal uses various pulse repetition frequencies (PRF) and is altered either intentionally or unintentionally. Measurement of the pulse repetition frequency provides information about the purpose of the radar. This forms an important step in electronic support (ES) receivers. The obtained information is further processed for emitter identification in radar intercept receivers. Modern radars are employed in secret operations during crisis and in electronic warfare. This increases the need for emitter identification having no prior knowledge of the transmitted waveform, to understand the radar's purpose. Emitter identification helps in identifying possible threats from enemy and thus forms a necessary first step to counter the enemy threat emitters [2]. Previous methods for emitter recognition include passing the sorted pulse train and a delayed version through a coincident circuit. Another method uses the time of arrival of the incoming pulses while another method using autocorrelation function is discussed in [8]. All these methods are computationally very complex and result in overloading of the processor. Blanking is used in emitter recognition in a dense environment where pulses related to a particular emitter are blanked once that emitter has been recognized. Most of the radar waveforms can be classified in 6 major modulations types either fully or piece by piece fashion namely constant, staggered, jittered, sliding, dwell and switch and periodic PRI [1]. These different PRIs can be described using the function

$$
F(n)=t_{n+1}-t_{n}=x_{n,} \text { for } n=1,2, \ldots N-1
$$

Where, $t_{n+1}-t_{n}$ defined the time of arrival and $x_{n}$ is the pulse repetition interval. Fig. 1 illustrates the same for all the six types of modulation PRIs. Jittered PRI here describes the random intentional variation of the PRI, and is different from unintentional PRI variation due to radar transmitters and receivers. 

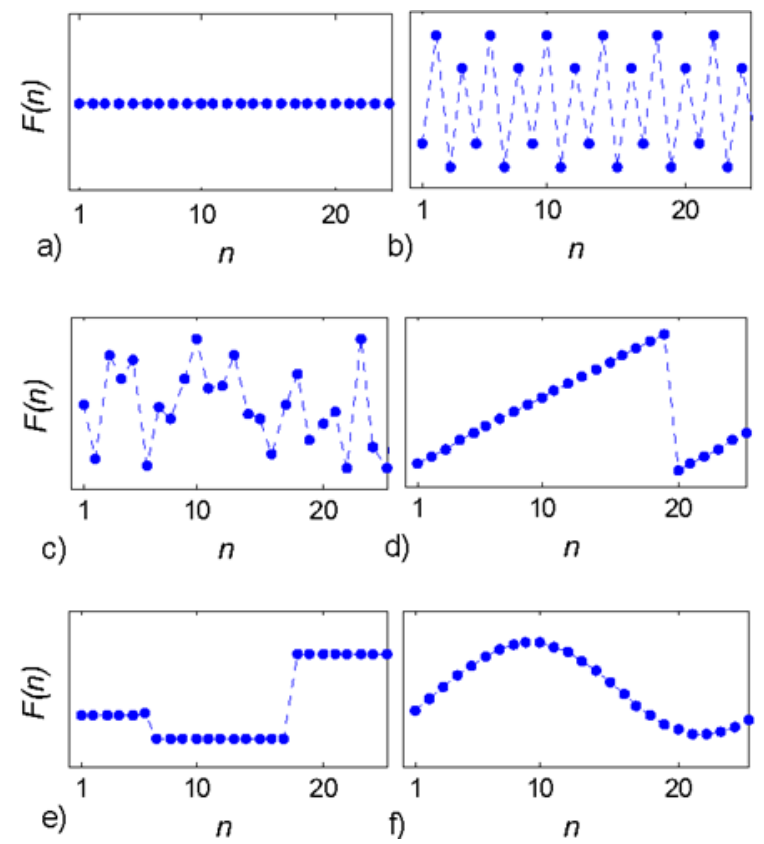

Figure 1a) Constant, b) Stagger, c) Jittered, d) Sliding, e) Dwell and Switch, and f) Periodic PRI.

PRI recognition is done by using statistical properties like a histogram. This method however requires a high number of pulses and also loses the sequential properties of the waveform. Another difficulty faced with histogram-based PRI recognition is with choosing the right detection threshold and bin width. Also, histogram-based methods can identify constant, staggered and jittered PRI. Waveform recognition based on the sequence of the PRI is also done in [3] using a neural network classifier.

This study aims to recognize all the mentioned PRI waveforms with arbitrary modulation parameters automatically. Here, 5 different features are chosen based on either statistical properties or the sequential properties of the waveform and a multi-layer perceptron (MLP) classifier is used. A pulse sorter is used along with time of arrival method and simulations are performed considering the noisy signal environment [6].

However, in practical radars, the PRI is often varied to overcome blind speed in moving target indicator (MTI) and for anti-jamming techniques. The discussed method deems unfit for use in case of variable PRI methods and in dense environments [2]. Thus, for emitter recognition, gate time interval method is discussed which works well for variable PRI as well as in dense environments. Also, the computational complexity for this method is less than the feature selection method using neural network.

\section{Feature Selection}

Feature selection refers to selecting a unique quantifier to differential the waveforms amongst themselves. These features should provide good separation capability for waveforms of different class while maintaining invariability with parameter variation within the same category. Also, the computation required to calculate the selected features should be less so as to maintain real-time emitter identification. 5 features are selected based on statistical and sequential information of the pulses and scaled between $0-1$.

\subsection{Features based on statistical properties}

Histogram-based PRI modulation recognition works well for constant, staggered and dwell and switch PRI but is complicated for periodic, jittered and sliding PRI as the histogram is based on the bin widths. The peak detection used in the histogram is very sensitive. 

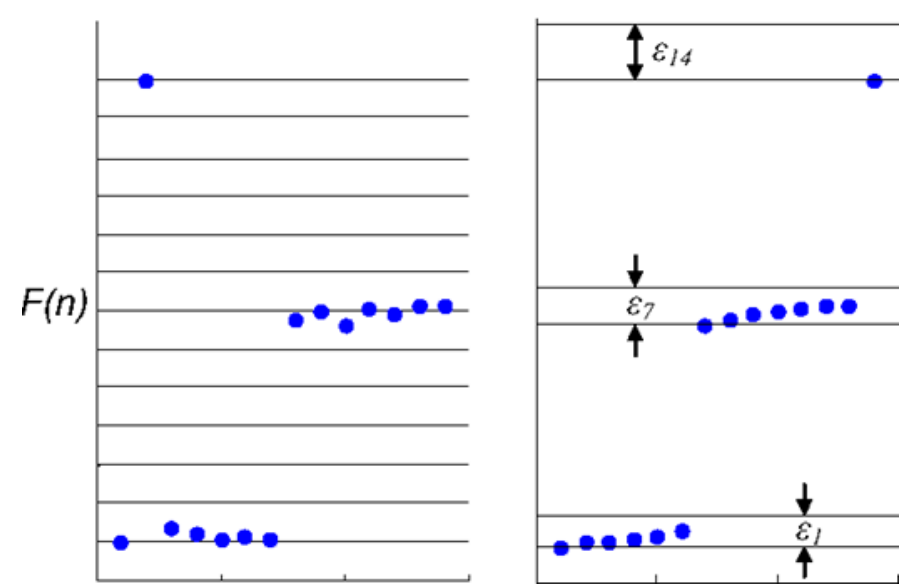

Figure 2: Removing the effect of unintentional jitter

The unintentional jitter causes the pulse peak to leak into adjacent bin widths. Thus, the new technique is used in which the histogram bins are defined to include the unintentional jitter given by:

$$
p_{n}=2 \cdot p \cdot x_{n}
$$

Where, $p$ represents the maximum expected unintentional jitter and $x_{n}$ is the bin width. In this method, the pulses are sorted in ascending order and pulses belonging to the respective bins including the tolerances are collected. Fig. 2 shows the traditional histogram and the updated histogram for dwell and switch PRI. Unintentional jitter is seen in fig. 2a. An outliner is also seen which is due to a missing pulse. While in fig. 2b, the pulses are sorted and leaked pulses are also collected based on the maximum expected unintentional jitter.

\section{Feature 1: Single histogram peak}

Single histogram peak is the ratio of second highest peak and the highest peak in the modified histogram. It defines the amount of constant PRI present and is given by:

$$
f_{1}=\frac{n_{\max -1}}{n_{\max }}
$$

Where, $n_{\max }$ is the highest peak and $n_{\max -1}$ is the second highest peak. The single histogram peak value for constant PRI is small. This is because the constant PRI consists of single strong peak in the histogram thus making $n_{\max }$ value very high and low $n_{\max -1}$. Thus, this feature is used to distinguish constant PRI waveform from other waveforms.

\section{Feature 2: Stable sum}

Stable sum uses the sequential difference histogram (SDIF) to calculate higher order histograms [7]. In a $k^{t h}$ level histogram, where $k$ is the difference level, consisting of $\mathrm{N}$ pulses, the number of intervals is given by

$$
M^{k}=N-k-1
$$

In this, the value of $k$ is varied and highest peak of these histograms are recorded. A high value of single peak as compared to others indicated presence of periodic pulse train. Thus, for this feature, the ratio of the highest peak to the total pulse intervals is calculated. This is given by:

$$
f_{2}=\max \left(N_{\max }^{k} / M^{k}\right) \quad k=2,3 \ldots k_{\max }
$$

Where, $k_{\max }$ is the highest difference level and $N_{\max }^{k}$ is the highest histogram value for given $k$. Stable sum is used to differentiate periodic waveforms from non-periodic waveforms. As seen from fig. [1] That staggered waveform is periodic while PRI in jittered waveform is random, hence, stable sum is used to differential between them.

\section{A. Features based on sequential information}


Histogram-based features lead to ambiguity while separating all the discussed PRI waveforms. Therefore, new features are discussed. These features are based on the sequential information of the pulses. Eq. 1 is differentiated resulting in second difference time of arrival (TOA) [5]

$$
z_{n}=x_{n+1}-x_{n}, \quad n=1,2, \ldots . N-2
$$

As the information required is the ordering of the pulses, therefore, above equation is transformed to a signum function as

$$
s=\operatorname{sgn}(z)
$$

Where,

$$
\operatorname{sgn}\left(z_{n}\right)=\left\{\begin{array}{cc}
-1 & \text { for } z_{n}<-\epsilon_{n} \\
0 & \text { for }\left|z_{n}\right| \leq-\epsilon_{n} \\
+1 & \text { for } z_{n}>\epsilon_{n}
\end{array}\right.
$$

Where, $\epsilon_{n}$ is the tolerance level for different bin widths

\section{Feature 3: Pulse interval changes}

Pulse interval changes is used to distinguish the stable pulse interval waveforms from those having continuous changes in pulse intervals. The feature is defined as the ratio of the amount of pulse interval changes with the number of pulses.

$$
f_{3}=\sum_{k=1}^{N-2} \frac{\left|s_{k}\right|}{N-2}
$$

Where, $s_{k}$ is the $k-t h$ element in vector $\mathrm{s}$

\section{Feature 4: Directional pulse interval change}

To distinguish the pulses in which the pulse interval change either increases or decreases, i.e., changes in one direction only, this feature is used. It is given by:

$$
f_{4}=\left|\sum_{k=1}^{N-2} S_{k}\right| /_{N-2}
$$

As seen from fig. 1, siding pulse waveform has pulse interval change in one direction only, increasing here, this feature is used to distinguish this waveform from others and has a high value for sliding PRI type waveform.

\section{Feature 5: Local extrema of pulse intervals}

In case, the pulse waveform has many local maxima and minima, this feature can be used. In this feature, the signum function is differentiated as:

$$
h_{k}=s_{k+1}-s_{k}, \quad k=1,2, \ldots \ldots N-3
$$

And the feature is given by:

$$
f_{5}=\sum_{k=1}^{N-3}\left|\operatorname{sgn}\left(h_{k}\right)\right|{ }_{N-3}
$$

Pulses with many local maxima and minima will have a higher value of this feature as compared to other pulses which do not have many local maxima and minima. As seen in fig. 1, staggered and jittered pulse waveform has many local maxima and minima, hence, is used to distinguish them from other waveform types.

\section{Limitations and Classification Capability}

It is assumed that the environment is constant during the observation period. Dwell and switch PRI type cannot be distinguished from constant PRI if less than one stage is simulated. Therefore, in case of Dwell and switch, two stages of stable pulse interval are simulated. Also, periodic PRIs having observation time less than one modulation period are not simulated. 

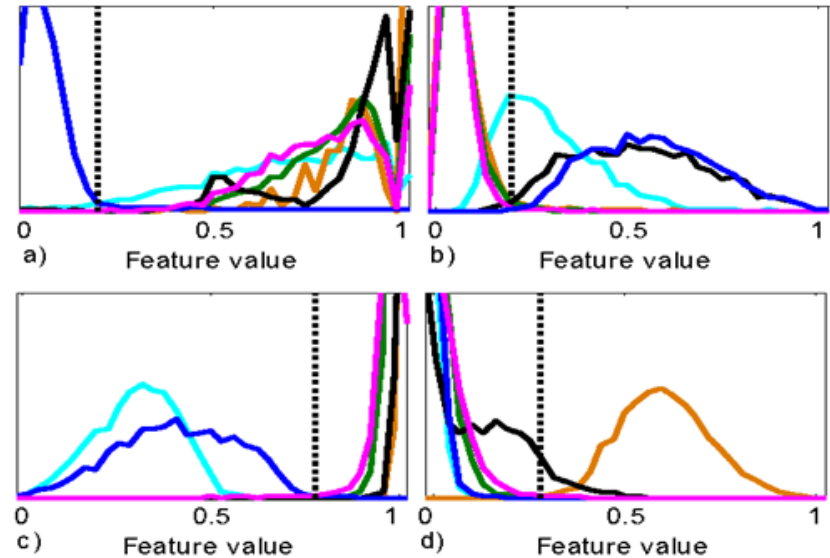

b)

Feature value

c) Feature value

d)
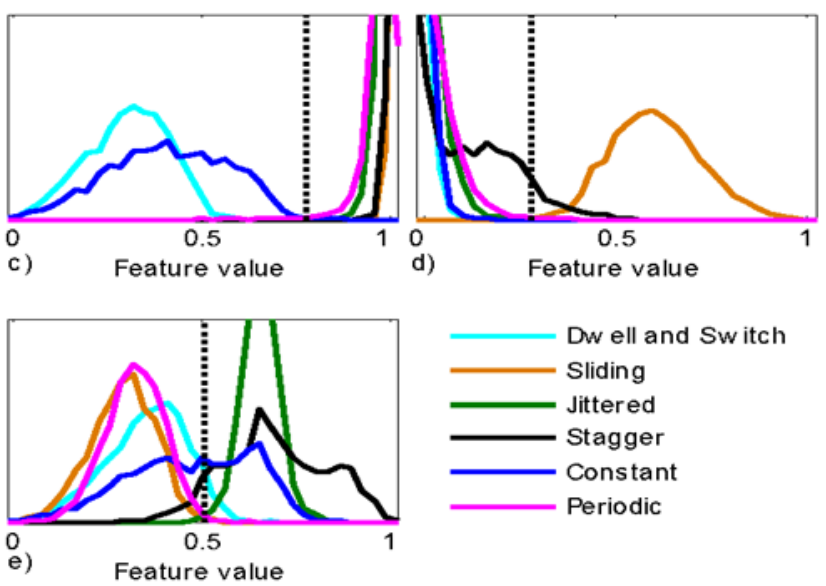

Figure 3: Features and their values for different

Fig. 3 shows the probability distribution for different features discussed above for all the mentioned waveforms. The features which do not overlap are able to distinguish the modulation type without any ambiguity.

\begin{tabular}{|l|l|l|l|l|l|}
\hline \multicolumn{7}{|c|}{ Feature } \\
\hline & 1 & 2 & 3 & 4 & 5 \\
\hline Constant & 0 & 1 & 0 & 0 & $\mathrm{X}$ \\
\hline Stagger & 1 & 1 & 1 & $\mathrm{X}$ & $\mathrm{X}$ \\
\hline Jittered & 1 & 0 & 1 & 0 & 1 \\
\hline Sliding & 1 & 0 & 1 & 1 & 0 \\
\hline $\begin{array}{l}\text { Dwell and } \\
\text { switch }\end{array}$ & 1 & $\mathrm{X}$ & 0 & 0 & $\mathrm{X}$ \\
\hline Periodic & 1 & 0 & 1 & 0 & 0 \\
\hline
\end{tabular}

Figure 4: Separation capability of the features

Table in figure 4 shows the performance of the features for different types of modulation PRI. The values given are 0,1 and $X$. The features with value 0 provide good separation from features with value 0 . And X essentially means it doesn't provide good feature separation. For eg, feature 1 has value 1 for all waveform type but constant PRI. Thus, this feature can be used to distinguish constant PRI type waveform [6]. Thus, it can be said that the selected features either individually or collectively can be used to identify the PRI modulation type and ultimately for emitter recognition.

\section{Gate Time Method}


In this method, the waveform data is first collected for the given observation period, $\mathrm{T}$, which is then divided into gate intervals, $\tau$. The number of pulses occurring in the given gate pulse is recorded. After this, the relative frequency of the pulses in the gate interval is calculated as shown:

$$
x_{t}=n_{t}-n_{\min }
$$

Where, $n_{t}$ is the number of pulses in gate interval $t$ and $n_{\min }$ is the minimum number of pulses recorded in any gate interval during the observation period. This recorded relative pulse gives information about the waveform type. Also, in the case of the dense environment, i.e. in the case of multiple emitters, the recorded pulses are found to be of the order of the number of emitters.

The discussed method is a probabilistic method where the probability of occurrence of states are found and compared. The computational complexity offered using this method is less compared to the TOA method [4]. Here, it is assumed that the environment is kept constant during the observation period and the pulses are unsynchronized to consider a realistic scenario. A case of fixed PRI and variable will be discussed in detail below.

\section{EMITTERS WITH FIXED PRI}

In case of fixed PRI, the gate time, $\tau$, is selected as:

$$
W z<\tau<(W+1) z
$$

Where, $z$ is the PRI and $W$ represents a non negative integer. Let us assume the time from gate pulse to pulse starting time be $\theta_{t}$. Now, consider a case where PRI is given by 1.2 and gate pulse as shown. In this case, the values are given in table

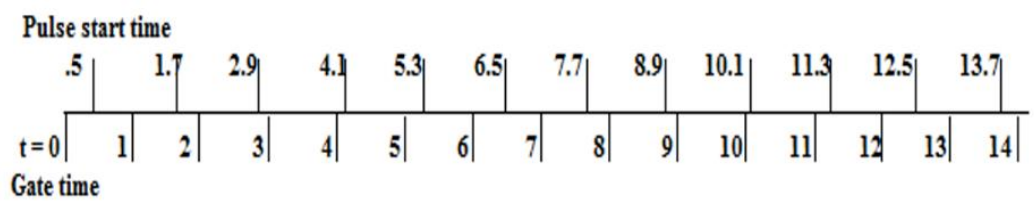

Figure 5: Gate time and pulse start time for fixed PRI

Thus, it can be seen that $x_{t}=0$ if $0<\theta_{t}<\tau-w z$ and $x_{t}=1$ if $\tau-w z<\theta_{t} \leq z$

\begin{tabular}{|c|c|c|}
\hline Gate interval & $\boldsymbol{\theta}_{\boldsymbol{t}}$ & $\boldsymbol{x}_{\boldsymbol{t}}$ \\
\hline $0-1$ & .5 & 1 \\
\hline $1-2$ & .7 & 1 \\
\hline $2-3$ & .9 & 1 \\
\hline $3-4$ & 1.1 & 0 \\
\hline $4-5$ & .1 & 1 \\
\hline $5-6$ & .3 & 1 \\
\hline $6-7$ & .5 & 1 \\
\hline $7-8$ & .7 & 1 \\
\hline $8-9$ & .9 & 1 \\
\hline $9-10$ & 1.1 & 0 \\
\hline $10-11$ & .1 & 1 \\
\hline $11-12$ & .3 & 1 \\
\hline $12-13$ & .5 & 1 \\
\hline $13-14$ & .7 & 1 \\
\hline & & \\
\hline
\end{tabular}

In this case, the states for $x_{t}$ is 0 and 1 with probabilities,

$$
p_{0}=\frac{(w+1) z-\tau}{z} \text { and } p_{1}=\frac{\tau-W z}{z}
$$




\section{Dense environment}

Considering K fixed PRI emitters, the state value is given by $x_{t}=\{0,1,2 \ldots . k\}$ where $k=N_{\max }-$ $N_{\min }$. In this case, the probabilities of the states $p_{j}^{k}$, where $\mathrm{j}$ is the state value and $\mathrm{k}$ is the number of emitters is given in terms of $p_{0}^{k}$ as:

$$
\begin{aligned}
p_{j, K}= & \sum_{i_{1}, i_{2}, \ldots, i_{j}=1}^{K}\left(1-p_{i_{1}}\right)\left(1-p_{i_{2}}\right) \ldots\left(1-p_{i_{j}}\right) p_{i_{j}}+1 p_{i_{j}}+2 \cdots p_{i_{K}} \\
& i_{1}<i_{2}<\ldots<i_{j} \\
& \left\{i_{j}+1, i_{j}+2, \ldots, i_{K}\right\}=\{1,2, \ldots, k\}-\left\{i_{1}, i_{2}, \ldots, i_{j}\right\} \\
& , j=1,2, \ldots, K .
\end{aligned}
$$

Thus, for fixed PRI, in case of dense environment, each possible state will have a fixed probability and thus can be identified using maximum likelihood by observing the sequence of $x_{t}$

\section{Emitters with Variable PRI}

In case of single emitter, the number of states can be more than 2 depending on the gate time and type of emitter. Using the similar approach as of fig. [4], The probabilities of different states for 2 stagger waveforms having PRI, $z_{1}$ and $z_{2}$ is found shown in fig. [5]. The gate time is considered to be less than both PRI and $z_{1}<z_{2}$

\begin{tabular}{|c|c|c|c|c|}
\hline Case & $\begin{array}{c}\text { Gate time }(\tau) 1 \text { imits } \\
W=0,1,2 \ldots\end{array}$ & $\begin{array}{c}\text { Possible } \\
\text { number } \\
\text { of } \\
\text { pulses }\end{array}$ & $\begin{array}{c}\text { States } \\
\text { of } \\
\text { model }\end{array}$ & Model state probabilities \\
\hline A & $W\left(z_{1}+z_{2}\right)$, & $2 W$ & 0 & $p_{0}=\frac{(2 W+1)\left(z_{1}+z_{2}\right)-2 \tau}{z_{1}+z_{2}}$ \\
\hline B & $W\left(z_{1}+z_{2}\right)+z_{1}$ & $2 W+1$ & 1 & $p_{1}=\frac{2 \tau-2 W\left(z_{1}+z_{2}\right)}{z_{1}+z_{2}}$ \\
\hline & $W\left(z_{1}+z_{2}\right)+z_{1}$, & $2 W$ & 0 & $p_{0}=\frac{W\left(z_{1}+z_{2}\right)+z_{2}-\tau}{z_{1}+z_{2}}$ \\
\hline & $W\left(z_{1}+z_{2}\right)+z_{2}$ & $2 W+1$ & 1 & $p_{1}=\frac{2 z_{1}}{z_{1}+z_{2}}$ \\
\hline & $W\left(z_{1}+z_{2}\right)+z_{2}$, & $2 W+1$ & 0 & $p_{2}=\frac{\tau-z_{1}-W\left(z_{1}+z_{2}\right)}{z_{1}+z_{2}}$ \\
\hline & $(W+1)\left(z_{1}+z_{2}\right)$ & $2 W+2$ & 1 & $p_{1}=\frac{2 \tau-(2 W+1)\left(z_{1}+z_{2}\right)}{z_{1}+z_{2}}$ \\
\hline
\end{tabular}

Figure 6: Probability of state for variable PRI

Similar results can be found for 3 stagger waveform and above and are discussed in [3]. The results of state probability for fixed PRI and 2 stagger variable PRI can be used to find the state probability for different PRI for different gate time intervals. And using the appropriate probability density function, the state probability of any PRI can be found. 


\begin{tabular}{|c|c|c|c|}
\hline $\begin{array}{l}\text { Type of } \\
\text { PRI } \\
\text { variation }\end{array}$ & $\tau<\mathrm{a}$ & $\tau=\tau_{1}=(\mathrm{a}+\mathrm{b}) / 2$ & $\tau=2 \tau_{1}=a+b$ \\
\hline $\begin{array}{l}\text { Fixed } \\
\text { PRI }\end{array}$ & $\begin{array}{l}\mathrm{p}_{0}=(\mathrm{z}-\tau) / \mathrm{z} \\
\mathrm{p}_{2}=0\end{array}$ & $\begin{array}{l}\mathrm{p}_{0}=0 \\
\mathrm{p}_{2}=0\end{array}$ & $\begin{array}{l}\mathrm{p}_{0}=0 \\
\mathrm{p}_{2}=0\end{array}$ \\
\hline $\begin{array}{l}\text { Two } \\
\text { position } \\
\text { stagger }\end{array}$ & $\begin{array}{l}p_{0}=\frac{z_{1}+z_{2}-2 \pi}{z_{1}+z_{2}} \\
p_{2}=0\end{array}$ & $\begin{array}{l}\mathrm{p}_{0}=\frac{\mathrm{z}_{2}-\mathrm{z}_{1}}{2\left(\mathrm{z}_{1}+\mathrm{z}_{2}\right)} \\
\mathrm{p}_{2}=\mathrm{p}_{0}\end{array}$ & $\begin{array}{l}\mathrm{p}_{0}=0 \\
\mathrm{p}_{2}=0\end{array}$ \\
\hline Uniform & $\begin{array}{l}\mathrm{p}_{0}=\frac{\mathrm{a}+\mathrm{b}-2 \tau}{\mathrm{a}+\mathrm{b}} \\
\mathrm{p}_{2}=0\end{array}$ & $\begin{array}{l}\mathrm{p}_{0}=\frac{\mathrm{b}-\mathrm{a}}{4(\mathrm{a}+\mathrm{b})} \\
\mathrm{p}_{2}=\mathrm{p}_{0}\end{array}$ & $\begin{array}{l}\mathrm{p}_{0}=\frac{\mathrm{b}-\mathrm{a}}{3(\mathrm{a}+\mathrm{b})} \\
\mathrm{p}_{2}=\mathrm{p}_{0}\end{array}$ \\
\hline Sinusoidal & $\begin{array}{l}p_{0}=\frac{a+b-2 \tau}{a+b} \\
p_{2}=0\end{array}$ & $\begin{array}{l}\mathrm{p}_{0}=\frac{\mathrm{b}-\mathrm{a}}{\pi(\mathrm{a}+\mathrm{b})} \\
\mathrm{p}_{2}=\mathrm{p}_{0}\end{array}$ & $\begin{array}{l}p_{0}=\frac{4(b-a)}{\pi^{2}(a+b)} \\
p_{2}=p_{0}\end{array}$ \\
\hline
\end{tabular}

Figure 7: Probabilities for PRI recognition and parameter identification

In the case of a dense environment, i.e. when many emitters are present, the estimation is similar as discussed above. The probabilities of different states are estimated, the polynomial coefficient can be estimated, which in turn helps in finding the number of emitters and emitter parameters like $\tau, z, a, b$

\section{PRI Type Recognition and Parameters Estimation}

Another important step in emitter identification is PRI recognition and estimation of parameters $(\tau, z)$. In this, the state probabilities are calculated considering:

$$
\tau<a, \tau=\frac{a+b}{2} \text { and } \tau=a+b .
$$

The calculated probabilities are compared with standard results shown in fig. [7] for PRI estimation. Once the type of PRI is estimated, which is then furthered into parameter estimation using maximum likelihood. It first checks for fixed PRI, then 2 position stagger and finally uniform PRI. It faces difficulty in estimating uniform and sinusoidal PRI variations.

\section{Adaptive Identification and Pulse Identification}

In identifying when any new emitter starts transmitting, it is assumed that the emitter starts or stops transmitting one by one. In this case, the polynomial before the change in emitter behavior is considered known and the polynomial after emitter state change is calculated. Then comparing both the polynomial, before emitter state change and after state change, the emitter type is recognized. In this way, the computational burden in greatly reduced.

Another important step is the detection of any new overlapping pulses. In this case, the IF signal is calculated and is seen that whenever a new pulse starts or stops, there is a sharp change in IF signal amplitude and phase. However, a detector to detect this change is very costly. Therefore, a combination of band pass filters and logic gate switch pulse detector is used. In this, if there are two overlapping pulses, it will be seen as two pulses at the pulse counter detector.

\section{Limitation and Detection Capability}

In emitter recognition using probabilistic method, for accurate estimates made from maximum likelihood, the zeroes of the polynomial should be real. Also, it is seen that correct estimates are made for a longer observation period. It is also seen that the value of $\tau$ for which the maximum value of discriminant of a polynomial is obtained, provides the best result for emitter recognition. However, it is seen that instead of finding the roots of the polynomial, the adaptive approach works better for PRI identification and estimating other parameters.

\section{Conclusion}


Waveform recognition or emitter recognition forms a challenging problem especially if time domain agility is present. Automatic recognition of waveform is essential to take timely measures. Here, recognition is done based on PRI modulation. 5 features are discussed based on statistical and sequential properties of the signal. A multi-layer perceptron neural network was formed for validating the separation performance of the features. It is seen that the selected features can distinguish very well between constant, dwell and switch, jittered, staggered, periodic, sliding-type PRIs. Hence, the selected features along with the application of neural networks work fine for emitter recognition. The discussed approach however doesn't work for variable PRI and in a dense environment.

The gate time method discussed above is an adaptive method and adapts itself with changes in the environment. Also, this method doesn't involve any searches for features and this provides a lower computational burden. Thus, this method allows emitter with time agility to be recognized with high accuracy even in a dense environment at a reasonable cost.

\section{Acknowledgments}

This work was supported partially by Research Council (EPSRC) in the UK under grant EP/E022936 and the European Union's Horizon 2020 research and innovation programme under grant agreement H2020-MSCA-ITN-2016 SECRET-722424.

\section{Reference:}

[1] Kauppi, J. P., \& Martikainen, K. S. (2007). An efficient set of features for pulse repetition interval modulation.

[2] Hawkes, R. M. (1980). Radar emitter recognition using pulse repetition interval. ELECTRONICS RESEARCH LAB ADELAIDE (AUSTRALIA).

[3] Noone, G. P. (1999, February). A neural approach to automatic pulse repetition interval modulation recognition. In 1999 Information, Decision and Control. Data and Information Fusion Symposium, Signal Processing and Communications Symposium and Decision and Control Symposium. Proceedings (Cat. No. 99EX251) (pp. 213-218). IEEE.

[4] Hawkes, R. M. (1980). Radar emitter recognition using pulse repetition interval. ELECTRONICS RESEARCH LAB ADELAIDE (AUSTRALIA).

[5] Zade, A. A. T., \& Pezeshk, A. M. (2016, November). Pulse repetition interval detection using statistical modeling. In Proceedings of the 8th International Conference on Signal Processing Systems (pp. 100-104).

[6] Norgren, E. (2019). Pulse repetition interval modulation classification using machine learning.

[7] Milojević, D. J., \& Popović, B. M. (1992, February). Improved algorithm for the deinterleaving of radar pulses. In IEE Proceedings F (Radar and Signal Processing) (Vol. 139, No. 1, pp. 98-104). IET Digital Library.

[8] Ahmadi, M., \& Mohamedpour, K. (2012). PRI modulation type recognition using level clustering and autocorrelation. American Journal of Signal Processing, 2(5), 83-91. 